\title{
IDEAS becoming reality on the roadmap for biomarker validation in Alzheimer's disease
}

Amyloid PET has made the dream of dementia specialists come true, enabling visualisation in vivo and with high accuracy of the amyloid deposits that Alois Alzheimer described as senile plaques more than a century ago. Until recently, such visualisation could only be appreciated post mortem-far too late to be of any practical use. As is often the case, enthusiasm on this technological advancement was followed by more sobering observations. A large proportion (20-30\%) of people aged 65 years and older who have intact cognitive function have a positive amyloid PET scan, making the exam more suitable to rule out than to rule in the disease (ie, a negative amyloid PET rules out Alzheimer's disease pathology as the cause of cognitive impairment, but a positive scan does not imply Alzheimer's disease pathology as the underlying factor). Additionally, amyloid is just one of two pathophysiological markers of Alzheimer's disease, the other being tau, which is more closely associated with symptom onset and for which PET tracers are emerging. In this apparently grim scenario, how can a diagnostic test of brain amyloidosis improve clinical outcomes?

In the Journal of the American Medical Association, Rabinovici and colleagues ${ }^{1}$ published the first results of the Imaging Dementia-Evidence for Amyloid Scanning (IDEAS) study, a large US-based study on amyloid PET for the diagnosis of Alzheimer's disease. They found that among Medicare beneficiaries evaluated by dementia specialists, the use of amyloid PET was associated with changes in clinical management within 90 days. These changes consisted mostly of prescription or discontinuation of drugs (Alzheimer's disease drugs; cognitive enhancers; drugs for mood, behaviour, and dementia risk factors; and drugs used to treat other neurological conditions) and counselling. Notably, a positive scan was followed by a marked increase in prescription of drugs for Alzheimer's disease, whereas practitioners rarely changed clinical management when the scan was negative. Impact on diagnostic confidence and drug prescription has been previously shown in smaller studies such as $\mathrm{ABIDE}^{2}$ and INDIA-FBP, ${ }^{3}$ although at a much smaller magnitude. The IDEAS authors acknowledge that the study does not provide an answer to whether the management changes are associated with improved clinical outcomes. The ongoing longitudinal component of IDEAS is collecting information about hospital admissions and mortality to address this issue and to explain why such a massive sample size $(n=16008)$ is needed.

The impact on health outcomes of diagnostic exams that allow identification of conditions amenable to a cure (eg, gastroscopy for gastric cancer and angiography for cerebral vessel occlusion) is obvious. However, the impact of diagnostic exams on conditions for which only palliative care exists is much subtler. Medicine is a highly integrated and non-deterministic discipline in which biomedical, psychological, psychosocial, and regulatory factors interact through multiple pathways. An uncertain diagnosis can trigger useless or misleading diagnostic exams, which can lead to ineffective or toxic drug interventions that can prompt inappropriate management choices, possibly producing adverse health outcomes. The impact on clinical outcomes of a more accurate diagnosis is affected by a number of stochastic factors that rigorous scientific method can barely measure and control (eg, the same diagnosis can have a range of different managements, and the same management can fit several diagnoses). Despite the hurdles, the Alzheimer's disease and dementia research community should strive to provide the best evidence for any medical intervention, be it diagnostic or treatment.

Whatever its pathophysiological role, brain amyloidosis is a major predictor of adverse cognitive outcomes in people without dementia, ${ }^{4,5}$ a necessary (although insufficient) feature in the current definition of Alzheimer's disease. ${ }^{6,7}$ IDEAS is collecting the much-needed hard evidence on the clinical validity and usefulness of an accurate and reliable amyloid detection tool such as amyloid PET, ${ }^{8}$ but one of its weaknesses is a noncontrolled design, as all patient underwent PET. A current European initiative, the AMYPAD diagnostic and patient management study, ${ }^{9}$ aims to add to the evidence by using a randomised design to study the impact of amyloid PET on diagnostic confidence and patient-related outcomes.

Although IDEAS and AMYPAD both focus on amyloid PET, dementia diagnostic tests make use of other biomarkers, such as metabolic fluorodeoxyglucose PET and CSF studies; CSF is more popular in (northern) Europe 
and allows the simultaneous assessment of amyloid $\beta 42$ and tau proteins. More initiatives examining the whole diagnostic armamentarium will be needed to develop evidence-based, cost-effective diagnostic investigations for patients with cognitive impairment and suspected Alzheimer's disease. However, coordinated and harmonised validation of biomarkers relying on different platforms such as PET and CSF will be a challenge. The Alzheimer's disease and dementia research community has developed a biomarker validation framework that should help to achieve precisely this goal. ${ }^{10}$

While waiting for drugs able to prevent or delay the progression of Alzheimer's disease, patients have the right to at least know what is affecting their memory, plan for the future, and receive the most appropriate pharmacological and non-pharmacological interventions available. IDEAS is a major step in this direction.

Giovanni B Frisoni, Isadora Lopes Alves, Frederik Barkhof Memory Clinic, University Hospital and University of Geneva, Geneva 1206, Switzerland (GBF); Department of Radiology and Nuclear Medicine, VU University Medical Center, Amsterdam, Netherlands (ILA, FB); and UCL Institute of Neurology and UCL Institute of Healthcare Engineering, University College London, London, UK (FB)

giovanni.frisoni@hcuge.ch

AMYPAD has received funding from the Innovative Medicines Initiative 2 Joint Undertaking under grant agreement number 115952. This Joint Undertaking receives the support from the European Union's Horizon 2020 research and innovation programme and European Federation of Pharmaceutical Industries and Associations. FB is supported by the National Institute for Health Research Biomedical Research Centre at University College London Hospital (UK).

GF and ILA declare no competing interests. FB reports board membership of Neurology, Brain, Radiology, MSJ, and Neuroradiology; personal fees from Springer, Bayer, and Biogen; personal fees from Roche, Apitope Ltd, IXICO Ltd, GeNeuro, and Novartis; and grants from Novartis, TEVA, Merck, Biogen, IMI-EU, GE Healthcare, UK MS Society, Dutch Foundation MS Research, NWO, and NIHR, outside the submitted work.

1 Rabinovici GD, Gatsonis C, Apgar C, et al. Association of amyloid positron emission tomography with subsequent change in clinical management among Medicare beneficiaries with mild cognitive impairment or dementia. JAMA 2019; 321: 1286-94.

2 de Wilde A, van der Flier WM, Pelkmans W, et al. Association of amyloid positron emission tomography with changes in diagnosis and patient treatment in an unselected memory clinic cohort: the ABIDE project. JAMA Neurol 2018; 75: 1062-70.

3 Boccardi $M$, Altomare $D$, Ferrari $C$, et al. Assessment of the incrementa diagnostic value of florbetapir F 18 imaging in patients with cognitive impairment: the Incremental Diagnostic Value of Amyloid PET With [18F]-Florbetapir (INDIA-FBP) study. JAMA Neurol 2016; 73: 1417-24

4 Vos SJ, Xiong C, Visser PJ, et al. Preclinical Alzheimer's disease and its outcome: a longitudinal cohort study. Lancet Neurol 2013; 12: 957-65.

5 Vos SJ, Verhey F, Frölich L, et al. Prevalence and prognosis of Alzheimer's disease at the mild cognitive impairment stage. Brain 2015 138: $1327-38$.

6 Dubois B, Feldman $\mathrm{HH}$, Jacova C, et al. Advancing research diagnostic criteria for Alzheimer's disease: the IWG-2 criteria. Lancet Neurol 2014; 13: 614-29.

7 Jack CR Jr, Bennett DA, Blennow K, et al. NIA-AA Research Framework: toward a biological definition of Alzheimer's disease. Alzheimers Dement 2018; 14: 535-62.

8 Clark CM, Schneider JA, Bedell BJ, et al. Use of florbetapir-PET for imaging $\beta$-amyloid pathology. JAMA 2011; 305: 275-83.

9 Frisoni GB, Barkhof F, Altomare D, et al. AMYPAD diagnostic and patient management study: rationale and design. Alzheimers Dement 2019; 15: 388-99.

10 Frisoni GB, Boccardi M, Barkhof F, et al. Strategic roadmap for an early diagnosis of Alzheimer's disease based on biomarkers. Lancet Neurol 2017; 16: 661-76. 